\title{
MMSE based beamformer in massive MIMO-IDMA downlink systems
}

\author{
Aasheesh Shukla ${ }^{1}$, Vishal Goyal ${ }^{1}$, Manish Kumar ${ }^{1}$, \\ Munesh Chandra Trivedi ${ }^{2}$, Vinay Kumar Deolia ${ }^{1}$
}

\begin{abstract}
Now-a-days Massive MIMO (mMIMO) become an attractive technology due to its spectral and energy efficiency by the means of simple signal processing. However, in overloaded scenario, wherein the number of users equipments (UEs) are larger than number of antennas, the spectral efficiency (SE) suffers and hence error rate performance, it has been shown recently that use of code domain NOMA in mMIMO can improve the SE performance. Further, interleave division multiple access (IDMA) has been drawn much attention as a suitable code domain non-orthogonal multiple access (NOMA) for future communication standards. This paper proposes the work in two folds, first a massive multiple input and multiple output (MIMO) and IDMA communication system is jointly proposed in which antennas on the base station serves users simultaneously in the same frequency band. Both and are large in numbers. Secondly, the minimum mean square error (MMSE) based beamformer is suggested to combat the propagation loss and effect of multiple access interference (MAI), for massive MIMO-IDMA system under downlink communication constraints. With the help of simulation results, the performance of the proposed system with MMSE beamforming have been investigated in terms of BER vs SNR curve plot.
\end{abstract}

K e y w or d s: massive MIMO, precoding, 5G, IDMA, MMSE

\section{Introduction}

The growing number of users and higher data rate services has crucially required the increased capacity and the quality for wireless communication. Based on data from $\mathrm{ABI}$ research, the mobile equipments may increase up to 49 million and internet of things (IOT) up to 26 billion by 2020 [1]. Hence, a robust communication system is inevitable for future communication requirements. Recently, mMIMO system, in which the base station is furnished with the antenna array of larger size than the active user equipments has attracted attention of researchers due to its improved spectral and energy efficiency [2].

In the recent discussions, which are based on random matrix theory demonstrated that the uncorrelated noise and fast fading effects vanishes, if the number of antennas grows massive in numbers in a single cell [2]. Further, the number of antennas at base stations are typically higher than the number of users antennas, hence the large number of degrees of freedom (DoF) exists and so the transmitted signal can be easily shaped in the way to minimize the interference [3]. Hence forth this can be said that mMIMO has potential advantages such as better energy efficiency with compared to single antenna system. However, in overload scenario wherein the number of UEs exceeds the number of base station antennas, then the spectral efficiency gets poor and hence the error rate performance of the mMIMO system. In recent research, this has been found that the use of code domain NOMA in mMIMO communication can improve the $\mathrm{SE}$, in the worst propagation conditions of UEs and hence error rate performance is also deteriorated [4].

Further, Interleave division multiple access (IDMA) has already been accepted as an efficient code domain non orthogonal multiple access (NOMA) scheme in 5G communication standards [5]. Code based NOMA schemes includes IGMA from Samsung, RDMA from Mediatek and IDMA from Nokia. These scheme are interleavingbased NOMA schemes, and originate from the fundamental concept of IDMA [6]. In Interleaving based NOMA (IDMA) scheme, interleaver patterns are utilizes to distinguish between different users. In the IDMA receiver, the low-complexity ESE algorithm is used and it provides log-likelihood ratio (LLR) estimates to decode the data [6-7].

Hence a robust communication system can be suggested by combining the massive MIMO and IDMA communication system. Although the combination of massive MIMO and NOMA has been already suggested in few research papers. However most of them considered the power domain NOMA [8].The gains are generally limited since power domain NOMA requires UEs with nonorthogonal channels, while core feature of mMIMO is to make all the UEs channel nearly orthogonal [8-9].

To improve the performance of mMIMO, this paper mainly focuses on the joint processing of code domain

\footnotetext{
1 Department of Electronics and Communication, GLA University, Mathura, India, aasheesh.shukla@gla.ac.in, 2 Department of Computer Science and Engineering, NIT, Agartala, India
} 


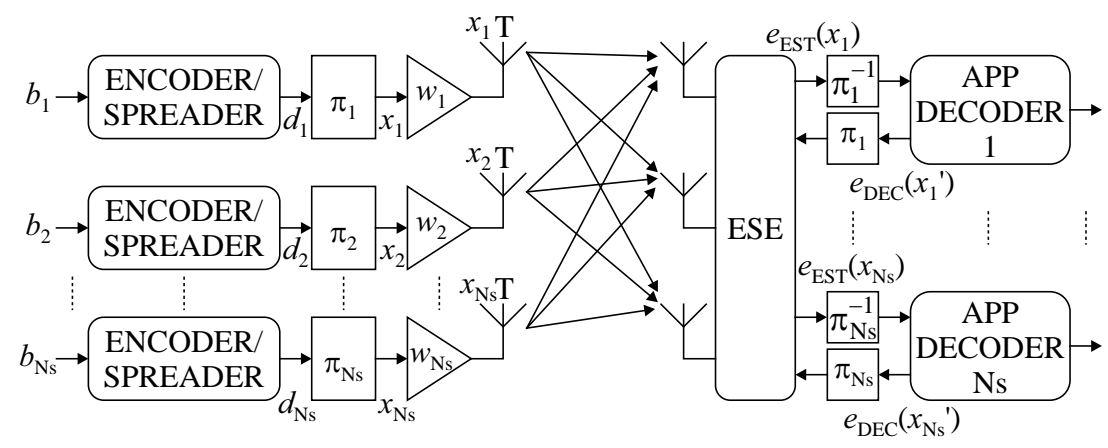

Fig. 1. Massive MIMO- IDMA system model

NOMA (IDMA) and m MIMO. In addition, the performance of the proposed system can be further enhanced with the use of transmit beamformers. The beamformers increase the gain of signal power in the desired user direction as well as sufficient attenuation is also achieved to unwanted users. Along with several beamforming algorithms, the chip level minimum mean-squared- error (MMSE) beamformer can be promptly used in spread spectrum systems (IDMA), in which the optimum beamforming weights are obtained by minimizing the mean square error between the spread signal and reference signal [10-12].

Hence, this paper clearly proposes mMIMO-IDMA system jointly, under downlink constraints $\left(N_{\mathrm{S}} \geq N_{\mathrm{R}}\right)$ to improve the SE and error rate performance of mMIMO. In addition the MMSE based beamforming scheme is used with proposed system to further improve the BER performance of system.

\section{Massive MIMO- IDMA system model}

A Massive-MIMO-coded NOMA (IDMA) communication system is considered with large antenna array $\left(N_{\mathrm{S}} \geq 64\right)$ to communicate with $N_{\mathrm{R}}$ single antenna users equipment (UE). The channel in between is considered as flat fading channel. With BPSK modulation and without any precoding, the received signal at time can be expressed as

$$
\boldsymbol{Z}[t]=\boldsymbol{H} \cdot \boldsymbol{x}^{\top}+\boldsymbol{n}[t], \quad t \in(1,2 \ldots N)
$$

where $\boldsymbol{H} \sim \mathbb{N}^{N_{\mathrm{S}} \times N_{\mathrm{R}}}(0,1)$ is a vector of fading channel coefficients, $\boldsymbol{n}[t] \sim N\left(0, \sigma_{n}^{2}\right)$ is a $N_{\mathrm{S}} \times 1$ Gaussian noise vector and $x=\left[x_{1}, x_{2}, \ldots, x_{N_{\mathrm{R}}}\right]$ is the transmitted message signal. Further at the transmitter side the information bits $b_{k}[t]$ are considered to be independent and identically distributed (iid) with symbol duration $T$. Similarly, the duration of $p_{k}[t]$ is called chip period $T_{\mathrm{c}}$ so the processing gain $N=T / T_{\mathrm{c}}$. The spreading waveform can be expressed as

$$
c_{k}[t]=\sum_{n=0}^{N-1} p\left[t-T_{\mathrm{c}} n\right] c_{k}[n]
$$

where $c_{k}[n]$ is spreading code, $p(t)$ is the impulse response of Nyquist pulse shaping filter. Assume that $K$ signals in downlink channel. So the signal spread for intended mobile user can be written as

$$
d_{k}(t)=\sum_{l=0}^{l=N-1} c_{k}(t-T l) b_{k}[l]
$$

this expresses the spread data waveform.

Further this can be interleaved by a $N$ length random interleaver $\Pi_{\ell}$ to obtain the transmission vector $\mathrm{x}_{\ell}=$ $\left[x_{\ell, 1}, x_{\ell, 2} \ldots x_{\ell, N}\right]^{\top}$.

Here, each $x_{\ell}$ is gaussianly distributed, ie $x_{\ell} \sim$ $N\left(0, \sigma_{x_{\ell}}^{2}\right)$ for $\forall \ell \in N_{\mathrm{S}}$ and received signal is already expressed in equation (1) [10]. Suppose that the weight vector of beamformer is $w_{k} \in\left[\begin{array}{lll}w_{1} & w_{2} \ldots \ldots \ldots \ldots w_{N_{\mathrm{S}}}\end{array}\right]$, then the transmitted signal from BS can be written as

$$
\chi=\sum_{k=1}^{N_{\mathrm{S}}} w_{k} \mathrm{H} x_{k}
$$

Further, the downlink communication is considered and perfect chip level synchronisation is assumed. The transmitted signal, presented in (4) is characterized by the standard channel coefficient $K^{(k)}$ for $k^{\text {th }}$ user.

At the receiver, the received signal is first correlated to spreading sequence and then de-interleave operation is performed. Hence, for standard MIMO multiuser communication channel, the received signal to desired $k^{\text {th }}$ user can be simply written as

$$
y_{(k)}=\left\{\sum_{k=1}^{N_{\mathrm{S}}} w_{k} \mathrm{H} x_{k}\right\}+\eta_{(k)}
$$

In the above equation, the channel noise is $\eta_{(k)} \sim \mathbb{N}(0,1)$ ie noise samples are gaussianly distributed with 0 mean and unit variance. $w_{(k)}$ is the MMSE beamformer weights.

\section{MMSE beamformer for Massive MIMO-coded NOMA}

Without the loss of generality, this can be assumed that the desired signal must interfere with other users, 
namely; multiple access interference and it should be reduced as much as possible to increase the quality of communication link. Beamforming can be the one way to combat the effect of MAI. In particular, MMSE beamforming is popular and have advantages over other beamforming schemes since it provides good bit-error rate (BER) performance for its performance metric while other beamforming techniques provides sum-rate computed from an idealized capacity equation [11-12]. MMSE beamforming can computes the best beamforming vectors by minimizing the error between the transmitted signal and received signal caused from both inter- users interference and noise. This makes the MMSE beamformer, easy to implement in practical systems.

It has also been shown that the MMSE beamforming achieves better system performance than other beamforming techniques in terms of both BER and sum-rate. Hence, in this paper, the MMSE beamforming is suggested for Massive MIMO- IDMA system. The weights of beamformer were obtained by minimising the difference of spread signal to the estimated signal at output. The weight update criterion can be expressed as

$$
\arg \left[\min E\|X-Y\|^{2}\right]
$$

where $X$ is the transmitted signal vector and estimated chip spread signal vector $Y$ of $k^{\text {th }}$ user. Further chip based MMSE based beamformer criterion can also be elaborated as

$$
J=\arg \left[\min E\|X-(W H X+\eta)\|^{2}\right]
$$

In the above equation the transmitter precoder (beamformer) can be represented by transformation matrix $\boldsymbol{W}$ . The total cost function and $L-2$ norm in expectation function can be further elaborated as

$$
\begin{gathered}
J=\arg \left[\min E\|X-(W H X+\eta)\|^{2}\right] \\
E=\| \begin{array}{l}
\mathrm{XX}^{\top}+\left({ }^{\top} H^{H} W W^{\top} H X+\eta \eta^{H}+2 X^{\top} H^{H} W \eta\right) \\
-2 X^{\top} H^{H} W \mathrm{X}-\mathrm{X}^{\top} \eta
\end{array}
\end{gathered}
$$

In (9), after taking the expectation of with respect to $\eta$ and $X$ respectively, and dropping the terms independent of $W,(9)$ can be reduced to

$$
J=H^{H} W W^{\top} H^{H}-2 H W
$$

By taking the derivative of $J$ with respect to the matrix $W$, and apply the minima condition to get the sufficient condition of beamforming weight update criterion;

$$
\frac{\partial}{\partial W}(J)=2 H^{H} W H-2 H=0 \Rightarrow W_{o p t}=H^{-1}
$$

\section{Performance analysis}

In this section the SINR performance of proposed system is presented. As we already discussed the optimum weights of MMSE beamformer in (11), so the received signal at the mobile receiver can be written as

$$
\begin{gathered}
y_{k}=\mathrm{x}_{k}+\text { interfering signal }+ \text { noise } \\
y(j)=\sum_{k=1}^{K} h_{k} x_{k}(j)+\eta(j) \mathrm{j} \in(1,2 \ldots \ldots . . \mathrm{J})
\end{gathered}
$$

here, this is assumed that the channel coefficient $h_{k}$ is priorily known to reciver. The noise samples are AWGN process ie $\eta \sim N\left(0, \sigma_{n}^{2}\right)$ and $\sigma^{2}=n_{0} / 2$. Due to the use of random interleaver the estimation can be done as chip by chip detection i.e. only one sample $y(j)$ at a time

$$
y(j)=h_{k} x_{k}(j)+\varsigma_{k}(j)
$$

Hence,

$$
\varsigma_{k}(j)=y(j)-h_{k} x_{k}(j)=\sum_{k^{\prime} \neq k} h_{k^{\prime}} x_{k^{\prime}}(j)+\eta(j)
$$

represents the interference plus noise in $y(j)$ with respect to user $k$. Further, with the help of the estimation of interference mean and variance the log likelihood ratio (LLR) can be written as

$$
e_{E S E}\left(x_{k}(j)\right)=2 h_{k} \frac{y(j)-\mathrm{E}\left(\varsigma_{k}(j)\right)}{\operatorname{var}\left(\varsigma_{k}(j)\right)}
$$

The average value of signal to noise ratio (SNR) for user-k can be expressed as

$$
\mathrm{SNR}=\frac{\mathrm{E}\left(\left|x_{k}(j)\right|^{2}\right)}{\mathrm{V}_{\varsigma_{k}}}
$$

where

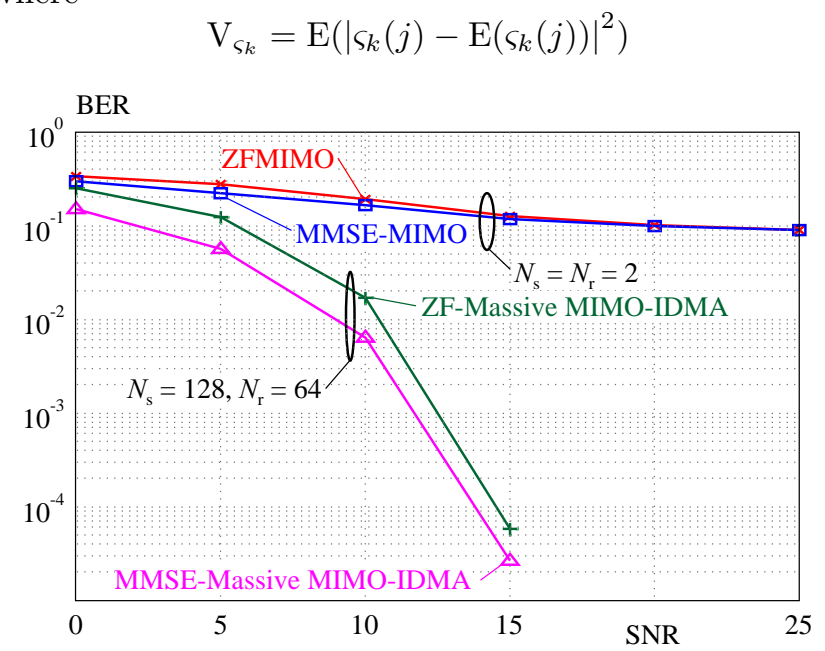

Fig. 2. BER performance of massive MIMO-IDMA system $N_{\mathrm{S}}=$ 128 and $N_{\mathrm{R}}=64$ 
With the help of (15) the average value of SNR can be calculated for user- $k$ and the optimum beamformer weights can lead to improved SNR as they derived with the help of (11). Further the BER performance can be defined as the function of SNR for user- $k$ $i e \mathrm{BER}=g(\mathrm{SNR})$. Now for the simulation purpose, the data bits $\left(b_{k}=1000\right)$ are being transmitted, after spreading $($ length $=16)$ and interleaved randomly $\pi_{k}$ in to $x_{k}$. The optimum beamforming vector is employed on the interleaved data to get the transmitted signal vector $i e x_{k}^{\top}$ Number of iteration $i t=10$ are used at the receiver to finally decide on the received bits. The iterative detection, based on turbo processing is used in the receiver. Further, the BER vs SNR performance curve is plotted to show the improvement of massive MIMO-IDMA system over MIMO system in downlink communication.

In the simulation experiment, the processing gain is taken as 16-th number of transmit and receive antennas are $\left(M_{S}=128\right.$ and $\left.N=64\right)$. The results are compared to simple MIMO system, in which transmit and receive antenna are kept same ie $N_{\mathrm{S}} 2, N_{\mathrm{R}}=2$. Figure 2 shows the simulation plot and clearly depicts the performance improvement of proposed system $i e$ in massive mMIMOIDMA the BER is of the order of $10^{-5}$ at the $15 \mathrm{~dB}$ SNR, whereas with MMSE-MIMO is at $10^{-1}$.

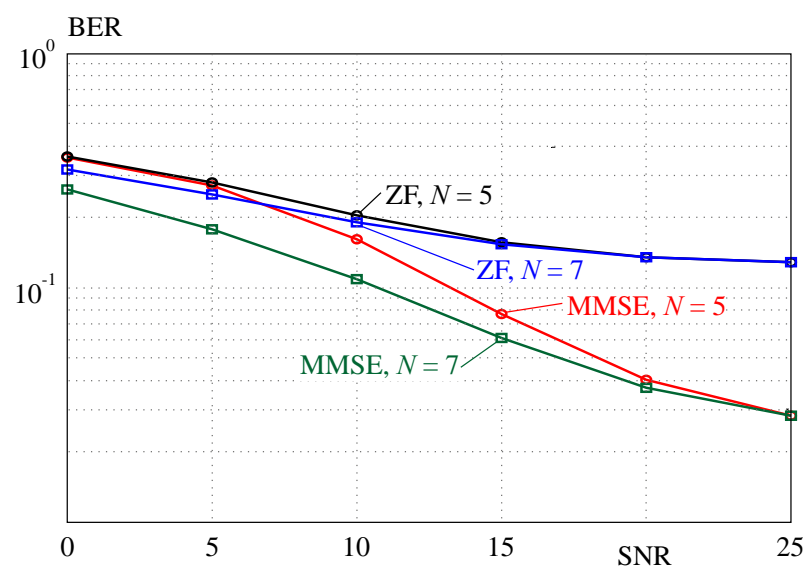

Fig. 3. SINR performance of massive MIMO-IDMA for $N=5,7$

In Fig. 3 the performance of massive MIMO-IDMA is illustrated for different number of users ie $N=5$ and $N=7$. This is clear from figure 3 that the performance of massive MIMO-IDMA with MMSE beamformer performs better in comparison to $\mathrm{ZF}$ beamforming algorithm for different number of users. The performance of proposed system is not detoriating with the increase in number of user at high value of SNR.

\section{Conclusion}

This paper presents the design of new Massive MIMOIDMA system for high data rate 5 th generation wire- less communications. Further MMSE beamforming is suggested for the proposed system to improve the SINR. The optimal condition for weight vector has been also derived using MMSE criterion. The simulation has been carried out to see the performance of the proposed system. Simulation results have demonstrated that the significant improvement have been observed in the BER performance of Massive MIMO-IDMA system with comparison to conventional 2X2 MIMO system. For the sake of simplicity, the random interleaving is used in proposed system However, in future work, the robust interleaving scheme as well as larger antenna array can be used to save memory requirement and better SNR respectively.

\section{REFERENCES}

[1] L. Liu, C. Yuen, Y. -L Guan, Y. Li, and C. Huang, "Gaussian Message Passing for Overloaded Massive MIMO-NOMA", IEEE transactions on wireless communication vol. 18, no.. 1, pp. 210-226, 2018 .

[2] T. L. Marzetta, "Non-cooperative cellular wireless with unlimited numbers of base station antennas" IEEE transactions on wireless communication, vol. 9, no. 11, pp. 3590-3600, 2010.

[3] C. Jinho and S. Perreau, "MMSE multiuser downlink multiple antenna transmission for CDMA systems", IEEE transactions on signal processing vol. 52, no. 6 pp. 1564-1573, 2004.

[4] T. P. Le-Mai, L. Sanguinetti, E. Björnson, and M.-G. Di Benedetto, "What is the Benefit of Code-domain NOMA in Massive MIMO?", 2019 IEEE 30th Annual International Symposium on Personal Indoor Mobile Radio Communications (PIMRC), pp. 1-5.

[5] W. Zhanji, K. Lu, C. Jiang, and X. Shao, "Comprehensive study comparison on 5G NOMA schemes", IEEE Access, pp. 18511-18519, 2018.

[6] L. Ping, L. Liu, K. Wu, and W. K. Leung, "Interleave division multiple access", IEEE Trans. Wireless Commun., vol. 5, no. 4, pp. 938-947, 2006.

[7] L. Ping, Interleave-division multiple access chip-by-chip iterative multi-user detection IEEE Commun, Mag., vol. 43 no. 6, pp. 519-523, 2005.

[8] L. Lei, C. Yuen, Y. -l Guan, Y. Li, and C. Huang, "Gaussian Message Passing for Overloaded Massive MIMO-NOMA", IEEE Transactions on Wireless Communications, vol. 18, no. 1, pp. 210-226, 2019

[9] M. N. Boroujerdi, S. Haghighatshoar, and G. Caire, "Low-complexity Statistically Robust Beamformer Design for Massive MIMO Systems", 22nd International ITG Workshop on Smart Antennas, 2018.

10] S. Aasheesh, V. Goyal, P. Mishra, and V. K. Deolia, "C", perative relay beamforming in IDMA communication networks, Journal of Electrical Engineering, vol. 69, no. 4, pp. 300-304, 2018.

[11] L. Lei, "Capacity-achieving MIMO-NOMA: iterative LMMSE detection", IEEE Transactions on Signal Processing vol. 67, no. 7, pp. 1758-73, 2019.

[12] K. Senel, H. V. Cheng, E. Bjornson, and E. G. Larsson, "What role can NOMA play in massive MIMO?", IEEE Journal of Selected Topics in Signal Processing vol. 13, no. 3, pp. 597-611, 2019.

Received 29 October 2019 